\title{
Program or Algorithm Execution Failure
}

National Cancer Institute

\section{Source}

National Cancer Institute. Program or Algorithm Execution Failure. NCI Thesaurus. Code C133625.

Problem associated with the failure of a program or algorithm to execute. Sudden /unexpected interruption to a program's execution. 